G lamblia; and in those aged betwęen 5 and 9 years $21 \%$ of specimens were positive. ${ }^{9}$ In Glasgow the results of stool examination were positive in $13.3 \%$ of Scots children, compared with $10.2 \%$ of Asian and only $1.1 \%$ of Chinese or African children. ${ }^{12}$ The higher incidence of giardiasis in the indigenous population was attributed to severe overcrowding being greatest among the local Scots.

Why some people develop symptoms with giardiasis, and others do not, is not understood. Severe symptoms may occur when only a few parasites are apparently present, whereas slight or no symptoms may be present in individuals with massive numbers of parasites. Differences in host susceptibility may thus be important. The finding of circulating antibody to $G$ lamblia $^{13}$ may indicate either that mucosal invasion in giardiasis is more common than has been appreciated or that it indicates increased mucosal permeability with resultant absorption of parasite antigen. The possibility of synergism between enterobacteria and $G$ lamblia causing damage to the intestinal mucosa cannot be excluded. ${ }^{14}$

The incubation period of symptomatic giardiasis is usually about two weeks (in contrast to the more common type of travellers' diarrhoea, which starts within a few days of arriving abroad) but may be some months. The main complaint is usually diarrhoea-explosive, watery, or loose stools which may be bulky and offensive and are often passed only in the mornings; blood and pus are absent. Other symptoms include weakness, abdominal distension and discomfort, anorexia, nausea, weight loss, flatulence, vomiting, belching, depression, and, in children, failure to thrive. The acute stage may last from a few days to several months. Some people may have subacute symptoms lasting for months or years. In a recent review of 40 patients with clinical giardiasis, ${ }^{14}$ symptoms had been present for up to five years. Twenty-three of these 40 patients had impaired xylose absorption, 20 had low vitamin $\mathrm{B}_{12}$ absorption, and 15 had steatorrhoea. More severe malabsorption was associated with more appreciable histological abnormalities of the ileal mucosa.

Giardiasis should be suspected in all patients presenting with diarrhoea starting one or more weeks after travel, or persisting for one or more weeks whether they have travelled or not. Parasitological confirmation of the diagnosis may be difficult because the presence of $G$ lamblia cysts or trophozoites may be irregular and unpredictable. ${ }^{15}$ Intermittent passage of parasites in the stool may be related to periods of active multiplication, ${ }^{2}$ and examination of stools passed on alternate days allows for this periodicity. The patient may have symptoms for over a week before the parasite becomes detectable. ${ }^{8}$ Stool examination using a concentration method will kill the trophozoites and so give a false-negative result if the stool contains these forms only (a comparative rarity in clinical practice, but direct examination of a fresh stool is needed to detect trophozoites). Delays of several months between the onset of symptoms and the diagnosis are common. ${ }^{7}$ Some workers prefer mucosal smear biopsy to stool examination, ${ }^{716}$ but this is bothersome and time consuming. ${ }^{2}$ Unfortunately, the more simple technique of sampling duodenal contents by the Enterotest or Duocaps capsule with nylon yarn ${ }^{17-19}$ is not generally available in Britain. But, if he suspects giardiasis, the wise physician will give a course of specific treatment regardless of laboratory findings.

A three-day course of metronidazole, $2 \mathrm{~g}$ once daily, produced a parasitological cure rate of $91 \%$, whereas a 10 -day course of mepacrine, $100 \mathrm{mg}$ thrice daily, eradicated the parasite in only $63 \% \cdot{ }^{14}$ But sometimes mepacrine is more successful than metronidazole. ${ }^{20}{ }^{21} \mathrm{~A}$ single dose of tinadazole,
$1 \mathrm{~g}^{22}$ or $2 \mathrm{~g}^{23}$ has produced high cure rates, but this drug is not yet generally available in Britain. Having treated an individual, the doctor should then check the other members of the household, for some or all of these may also be infected. ${ }^{6}$

${ }^{1}$ British Medical fournal, 1974, 2, 347.

2 Wolfe, M S, fournal of the American Medical Association, 1975, 233, 1362.

${ }^{3}$ Schultz, M G, fournal of the American Medical Association, 1975, 233, 1383.

4 Hartong, W A, and Arvanitakis, C, fournal of the American Medical Association, 1977, 237, 1078.

${ }^{5}$ Simpson, G E C, Medical fournal of Australia, 1975, 1, 601.

${ }^{6}$ Willcox, M, British Medical fournal, 1975, 3, 101.

7 Eastham, E J, et al, Lancet, 1976, 2, 950.

${ }^{8}$ Jokiph, A M M, and Jokiph, L, Lancet, 1977, 1, 1095.

${ }^{9}$ Meuwissen, J H E T, et al, Lancet, 1977, 2, 32.

${ }_{10}$ Ridley, D S, Fournal of Clinical Pathology, 1974, 27, 435.

11 Vella, E E, Lancet, 1977, 2, 33.

12 Goel, K M, et al, British Medical fournal, 1977, 1, 676.

${ }^{13}$ Ridley, M J, and Ridley, D S, Fournal of Clinical Pathology, 1976, 29, 30.

${ }^{14}$ Wright, S G, et al, Gut, 1977, 18, 343.

${ }^{15}$ Danciger, M, and Lopez, M, American Fournal of Tropical Medicine and Hygiene, 1975, 24, 237.

${ }^{16}$ Kamath, K R, and Murugasu, R, Gastroenterology, 1974, 66, 16.

17 Beal, C B, et al, American fournal of Tropical Medicine and Hygiene, 1970, $19,349$.

18 Thomas, G E, et al, South African Medical fournal, 1974, 48, 2219.

19 Palmer, R C, fournal of the American Medical Association, 1977, 237, 1078.

${ }^{20}$ Bassily, S, et al, fournal of Tropical Medicine and Hygiene, 1970, 73, 15.

${ }^{21}$ Babb, R R, et al, fournal of the American Medical Association, 1971, 217, 1359.

22 Salih, S Y, and Abdalla, R E, fournal of Tropical Medicine and Hygiene, $1977,80,11$.

${ }^{23}$ Pettersson, T, British Medical fournal, 1975, 1, 395.

\section{Ebola virus infection}

Some important questions raised by the outbreak last autumn in Southern Sudan and Zaire of a severe Marburg-fever-like illness need to be answered. What was the disease? What was its clinical picture and death rate? How did the outbreak start, spread, and finish-and why? What are the implications for control and containment of future infections? Finally, what should the doctor look for among patients with fever arriving in Britain or other non-tropical countries? We now have some facts about the outbreak and can answer some of these questions. ${ }^{12}$

The disease was caused by a virus morphologically indistinguishable from, but immunologically separate from, the Marburg virus-which was first isolated during the outbreak in that city in 1967 and later from the South African cases in 1975. It has been called Ebola virus from the name of the river and district initially affected in the recent outbreak.

Clinically the illness was severe, with a death rate of about half. The incubation period was from four to 16 days, after which patients had fever, headache, pains in the limbs and back, and then usually variable diarrhoea and vomiting. Pharyngitis and a dry cough were other common features. On the fifth day of the illness a rash appeared, as did a tendency to bleed from the gums, needle puncture sites, and elsewhere; jaundice was not seen. In pregnant women abortion and massive uterine haemorrhage were common. Those who died did so between the fourth and tenth day and in those who survived recovery was slow. Treatment was mostly supportive, but the reports suggest that immune plasma may be of benefit and interferon has been tried.

Epidemiologically, the outbreak appears to have started with a traveller, but how he contracted it is unknown. He trans- 
mitted the illness to several people with whom he had been in close contact, and after one patient had been admitted to Maridi hospital many further cases developed. Of the hospital's 230 staff, 76 developed the infection and 41 died-many of them at a time when the disease was thought to be typhoid fever and strict containment measures were not in operation. Transmission by unsterilised syringes and needles may have been responsible for many cases.

The outbreak in Zaire, about $1500 \mathrm{~km}$ distant from the Sudanese focus, developed three weeks later. There were 237 cases and 211 deaths, but the attack rate was only between 1 and 8 per 1000 exposed. The disease was thus not as highly transmissible as was at first thought. Very close contact was required, particularly with body fluids, while nursing patients at home or in hospital; droplet transmission seems not to have occurred.

The reservoir of infection is suspected to be rodents, and after the initial human infection the disease is transmitted by human contact. The infection can be contained by strict isolation, use of protective clothing, and adequate disposal of secretions and excretions. As soon as these measures were enforced during the recent outbreaks, new cases ceased to occur.

In future, this infection, together with Lassa fever, must be suspected as a cause of fever in severely ill persons either living in Central Africa or who have visited the region within the preceding two to three weeks, and in whom malaria can be excluded. Screening of such patients, in properly equipped centres and by trained staff, is essential. Last year one of the staff of the Microbiological Research Unit at Porton working with the virus accidentally inoculated himself, and the course of his illness is described in detail at $p$ 541. To control future outbreaks WHO is building up a register of centres and institutions with the necessary skills and personnel. to help at short notice. Finally, WHO itself has an important part to play in co-ordinating, providing, and exchanging information on this new illness, which poses yet another conundrum for the practitioner working in temperate climates.

\footnotetext{
1 World Health Organisation, Weekly Epidemiological Record, 1977, 52, 177.
}

2 World Health Organisation, Weekly Epidemiological Record, 1977, 52, 185.

\section{Management of oesophageal perforation}

Perforation of the oesophagus is a serious condition which is frequently fatal, particularly when diagnosis or surgical treatment is delayed. Contamination of the mediastinum with gastric secretions, food, and bacteria causes cardiorespiratory embarrassment, fluid loss, shock, and fulminating infection. Nevertheless, with prompt diagnosis and aggressive surgical treatment many patients can be saved.

An excellent retrospective review by Triggiani and Belsey ${ }^{1}$ of 110 cases of oesophageal perforation seen in one unit over 25 years highlights some important aspects of its management. During this period, 11 patients with spontaneous rupture were treated, their ages ranging from 28 to 75 . The commonest early symptoms were acute chest pain or epigastric pain, associated with dyspnoea after vomiting or retching. The most important diagnostic sign was surgical emphysema in the neck, which was present in every patient on admission. A chest radiograph and an oesophagogram (preferably with iodised oil) proved invaluable in confirming the diagnosis and locating the site of rupture. The ideal treatment of spontaneous rupture is primary repair provided it is done within 12 hours before the onset of suppurative mediastinitis. In the presence of established mediastinitis, severe dental sepsis, or oesophageal oedema, exteriorisation of the oesophagus followed by staged reconstruction offer the best hope of saving the patient's life, for half of those treated with conservative management (drainage of the pleural cavity and systemic antibiotics) will die.

Triggiani and Belsey saw 15 cases of instrumental perforation of the oesophagus. In eight patients this had occurred during diagnostic examination (affecting the cervical oesophagus in three and the lower oesophagus in three), and in seven during the dilatation of a stricture. In all cases the surgeon had used a rigid open-ended oesophagoscope, while perforation was commoner in patients under general rather than local anaesthesia. Wider use of the flexible fibreoptic oesophagogastroscope should reduce the incidence of perforation during diagnostic procedures. Such instruments should be included in every oesophago-gastric surgeon's armament and should not be the sole property of endoscopic physicians, who are unable to deal with the complications they cause.

Treatment of instrumental perforation depends on the site, the underlying lesion, and the speed of diagnosis. Perforations of the cervical oesophagus may be treated conservatively-no oral feeding, but intravenous alimentation and antibiotics. Perforations of the intrathoracic oesophagus, on the other hand, require immediate thoracotomy, repair of the perforation, and surgical treatment of the lesion for which the examination was performed, and the only delay should be for emergency resuscitation. The length of interval between the perforation and surgical treatment greatly influences the outcome: thus the death rate increases fourfold when treatment is delayed for over 24 hours. $^{2}$

Another fact reported in this review is that postoperative anastomotic fistulae occurred in 78 out of 2950 patients undergoing oesophageal surgery $(2.6 \%),{ }^{1}$ being the commonest cause of death. The incidence of fistulae varied according to the procedure: it was $12 \%$ after intrathoracic oesophagogastrostomy, $6 \%$ after cervical oesophago-gastrostomy, and $1 \%$ after interposition of left colon. The high incidence of leaks after the first procedure was ascribed to autodigestion of oesophageal tissue at the suture line due to the seepage of gastric secretions under the influence of negative intrathoracic pressure.

The only hope of saving the life of a patient with an established intrathoracic leak was found to be aggressive surgery. ${ }^{1}$ This consisted of exteriorisation of the remaining oesophagus, closure of the stomach and its return to the abdomen, cervical oesophagostomy, feeding gastrostomy, and staged reconstruction at a later date using the left colon. This policy, however, would appear justified only in patients with a good prognosis. For neglected or undiagnosed intrathoracic fistulae in which the patient's condition is critical, pleural drainage together with hyperalimentation is all that is usually possible. As in other forms of oesophageal perforation, the use of an iodised oil oesophagogram proves invaluable in the early diagnosis of postoperative leaks.

\footnotetext{
1 Triggiani, E, and Belsey, R, Thorax, 1977, 32, 241.

2 Sawyers, J L, et al, Annals of Thoracic Surgery, 1975, 19, 233.
} 